Vasile Ene, Ovidius University Constanţa, Romania Current address: 23 August 8717, Jud. Constanţa, Romania e-mail: ene@univ-ovidius.ro

\title{
LOCAL SYSTEMS AND TAYLOR'S THEOREM
}

\author{
Abstract \\ In this article we generalize Taylor's theorem, using the local systems \\ introduced by B. S. Thomson in [8].
}

We shall denote by $\mathcal{C}$ the class of all continuous functions, by $\mathcal{D}$ the class of all Darboux functions, by $\mathcal{B}_{1}$ the class of all Baire one functions, and by $\mathcal{D} \mathcal{B}_{1}$ the class of all Darboux Baire one functions.

Definition 1 (Thomson). ([8], p. 3). A family $\mathcal{S}=\{\mathcal{S}(x)\}_{x \in \mathbb{R}}$ is said to be a local system if each $\mathcal{S}(x)$ is a collection of sets with the following properties:

(i) $\{x\} \notin \mathcal{S}(x)$;

(ii) If $\sigma_{x} \in \mathcal{S}(x)$ then $x \in \sigma_{x}$;

(iii) If $\sigma_{x} \in \mathcal{S}(x)$ and $\sigma_{x} \subset A$ then $A \in \mathcal{S}(x)$;

(iv) If $\sigma_{x} \in \mathcal{S}(x)$ and $\delta>0$ then $\sigma_{x} \cap(x-\delta, x+\delta) \in \mathcal{S}(x)$.

Definition 2. Let $\mathcal{S}=\{\mathcal{S}(x)\}_{x \in \mathbb{R}}$ and $\mathcal{S}^{\prime}=\left\{\mathcal{S}^{\prime}(x)\right\}_{x \in \mathbb{R}}$ be local systems and let $x \in \mathbb{R}, A \subset \mathbb{R}$.

- (Thomson, [8], p. 5) We define the following local system: $\mathcal{S} \wedge \mathcal{S}^{\prime}=$ $\left\{\left(\mathcal{S} \wedge \mathcal{S}^{\prime}\right)(x)\right\}_{x \in \mathbb{R}}$, where $\left(\mathcal{S} \wedge \mathcal{S}^{\prime}\right)(x)=\mathcal{S}(x) \cap \mathcal{S}^{\prime}(x)$ (it is easy to verify that this is a local system).

- (Thomson, [8], p. 37). $\mathcal{S}$ is said to be bilateral at $x$ if $\sigma_{x}$ has $x$ as a bilateral accumulation point, whenever $\sigma_{x} \in \mathcal{S}(x)$. $\mathcal{S}$ is bilateral on $A$ if it is bilateral at each point of $A$.

Key Words: local systems, $\mathcal{D}, \mathcal{B}_{1}, \mathcal{D B}_{1}, u C M$, Taylor's Theorem

Mathematical Reviews subject classification: 26A24; 26A21; 26A15

Received by the editors October 10, 1995 
- (Thomson, [8], p. 18). Let $\mathcal{S}_{\infty}=\left\{\mathcal{S}_{\infty}(x): x \in \mathbb{R}\right\}$ denote the local system defined at each point $x$ as $\mathcal{S}_{\infty}(x)=\{\sigma: \sigma$ contains $x$ and has $x$ as an accumulation point $\}$. We can define right and left versions of this, by writing: $\mathcal{S}_{\infty}^{+}(x)=\{\sigma: \sigma$ contains $x$ and has $x$ as a right accumulation point $\}$ and $\mathcal{S}_{\infty}^{-}(x)=\{\sigma: \sigma$ contains $x$ and has $x$ as a left accumulation point $\}$.

- Let $\mathcal{S}_{\infty, \infty}=\mathcal{S}_{\infty}^{+} \wedge \mathcal{S}_{\infty}^{-}$. Clearly $\mathcal{S}_{\infty, \infty}(x)=\{\sigma: \sigma$ contains $x$ and has $x$ as a bilateral accumulation point $\}$.

- $\mathcal{S}$ is said to be $\mathcal{S}^{\prime}$-filtering at $x$ if $\sigma_{x}^{\prime} \cap \sigma_{x}^{\prime \prime} \in \mathcal{S}^{\prime}(x)$ whenever $\sigma_{x}^{\prime}, \sigma_{x}^{\prime \prime} \in \mathcal{S}(x)$. $\mathcal{S}$ is said to be $\mathcal{S}^{\prime}$-filtering on $A$ if it is so at each point of $A$.

- $\mathcal{S}$ is said to be filtering at $x$ if $\mathcal{S}$ is $\mathcal{S}$-filtering at $x$ (this is in fact Thomson's definition of [8], p. 10).

Remark 1. If $\mathcal{S}$ is $\mathcal{S}_{\infty, \infty}$-filtering on a set $A$ then it is a bilateral local system on $A$.

Definition 3. Let $\mathcal{S}=\{\mathcal{S}(x)\}_{x \in \mathbb{R}}$ be a local system. Let $F:[a, b] \rightarrow \mathbb{R}$ and $t \in[a, b] . \quad F$ is said to be $\mathcal{S}$-continuous at $t$ if for every $\epsilon>0$ there exists $\sigma_{t} \in \mathcal{S}(t)$ such that $|F(x)-F(t)|<\epsilon$, whenever $x \in \sigma_{t} \cap[a, b] . F$ is said to be $\mathcal{S}$-continuous on a set $A \subset[a, b]$ if it is so at each point $t \in A$.

Remark 2. For $t \in(a, b)$, Definition 3 is a reformulation of Thomson's Definition 31.1 of [8] (p. 70). However, our definition considers $t \in[a, b]$.

Lemma 1. Let $\mathcal{S}=\{\mathcal{S}(x)\}_{x \in \mathbb{R}}$ be a local system $\mathcal{S}_{\infty, \infty}$-filtering. Let $F$ : $[a, b] \rightarrow \mathbb{R}$ and $t \in[a, b]$. Suppose that there exists $c \in \overline{\mathbb{R}}$ with the following property: for every neighborhood $U_{c}$ of $c$ there is a set $\sigma_{t} \in \mathcal{S}(t)$ such that $(F(x)-F(t)) /(x-t) \in U_{c}$, whenever $x \in \sigma_{t} \cap[a, b]$ and $x \neq t$. Then the number $c$ is unique.

Proof. Suppose that there exists a number $d, d \neq c$, with the same properties as $c$. Let $U_{c}$ and $U_{d}$ be neighborhoods for $c$ respectively $d$ such that $U_{c} \cap U_{d}=\emptyset$. Let $\sigma_{t}^{\prime}, \sigma_{t}^{\prime \prime} \in \mathcal{S}(t)$, such that $(F(x)-F(t)) /(x-t) \in U_{c}$, whenever $x \in \sigma_{t}^{\prime} \cap[a, b]$, $x \neq t$, and $(F(y)-F(t)) /(y-t) \in U_{d}$, whenever $y \in \sigma_{t}^{\prime \prime} \cap[a, b], y \neq t$. Since $\mathcal{S}$ is $\mathcal{S}_{\infty, \infty}$-filtering it follows that $\sigma_{t}^{\prime} \cap \sigma_{t}^{\prime \prime} \backslash\{t\} \neq \emptyset$, a contradiction.

Definition 4. Let $\mathcal{S}=\{\mathcal{S}(x)\}_{x \in \mathbb{R}}$ be a local system $\mathcal{S}_{\infty, \infty}$-filtering. Let $F:[a, b] \rightarrow \mathbb{R}$ and $t \in[a, b]$.

(1) We denote the unique number $c$ of Lemma 1 by $\mathcal{S} D F(t)$ (the $\mathcal{S}$-derivative of $F$ at $t)$. 
(2) The function $F$ is said to be $\mathcal{S}$-derivable on $[a, b]$ if $\mathcal{S} D F(t)$ exists and is finite at each $t \in[a, b]$.

(3) If $F$ is $\mathcal{S}$-derivable on $[a, b]$ and the $\mathcal{S}$-derivative of $\mathcal{S} D F$ exists (finite or infinite) at $t$ then we denote this derivative by $\mathcal{S} D F^{(2)}(t)$.

(4) $F$ is said to be $\mathcal{S}^{(2)}$-derivable on $[a, b]$ if $\mathcal{S} D F^{(2)}(t)$ exists and is finite at each $t \in[a, b]$.

(5) Inductively we may define $\mathcal{S} D F^{(i)}(t)$ and the $\mathcal{S}^{(i)}$-derivability on $[a, b]$, $i=1,2, \ldots$. Let $\mathcal{S} D F^{(0)}(t)=F(t)$.

Remark 3. For $t \in(a, b)$, Definition $4,(1)$ is a reformulation of a part of Definition 7.1 of [8] (p. 14). Of course, Definition 4, (1) is less general, because Thomson's definition does not impose any conditions on the local system. However, our definition considers $t \in[a, b]$.

Lemma 2. Let $\mathcal{S}=\{\mathcal{S}(x)\}_{x \in \mathbb{R}}$ be a local system $\mathcal{S}_{\infty, \infty}$-filtering. Let $F$ : $[a, b] \rightarrow \mathbb{R}$. If $F$ is $\mathcal{S}^{(i)}$-derivable on $[a, b]$ then $\mathcal{S} D F^{(i-1)}$ is $\mathcal{S}$-continuous on $[a, b], i=1,2, \ldots$.

Definition 5. We define the following local systems:

- $\mathcal{S}_{1,1}=\left\{\mathcal{S}_{1,1}(x)\right\}_{x \in \mathbb{R}}$, where $\mathcal{S}_{1,1}(x)=\left\{S: x \in S\right.$ and $\underline{d}_{+}^{i}(S, x)=$ $\left.\underline{d}_{-}^{i}(S, x)=1\right\}$. (Here $\underline{d}_{+}^{i}$ and $\underline{d}_{-}^{i}$ are the interior right respectively left densities of $S$ at $x$ - see for example [8], p. 22). Let $F_{a p}^{(i)}(x)=$ $\mathcal{S}_{1,1} D F^{(i)}(x)$.

- For $\alpha, \beta \in(0,1)$, let $\mathcal{S}_{\alpha, \beta}=\left\{\mathcal{S}_{\alpha, \beta}(x)\right\}_{x \in \mathbb{R}}$, where $\mathcal{S}_{\alpha, \beta}(x)=\{S: x \in S$ and $\left.\underline{d}_{-}^{i}(S, x)>\alpha, \underline{d}_{+}^{i}(S, x)>\beta\right\}$. Let $F_{p r}^{(i)}(x)=\mathcal{S}_{\frac{1}{2}, \frac{1}{2}} D F^{(i)}(x)$.

Remark 4. The $\mathcal{S}_{1,1}$ and $\mathcal{S}_{\alpha, \beta}$ local systems are slight modifications of some systems introduced in [6] (pp. 81, 85), [7] (I, p. 75, 76) and [2] (p. 99).

Definition 6 (Preiss). ([5] or [3], p. 35). Let $F:[a, b] \rightarrow \mathbb{R}$. F is said to be lower internal ${ }^{*}$, if $F(x+) \geq F(x)$, whenever $x \in[a, b)$ and $F(x+)$ exists, and $F(x-) \leq F(x)$, whenever $x \in(a, b]$ and $F(x-)$ exists. $\mathrm{F}$ is said to be upper internal ${ }^{*}$ if $-F$ is lower internal* ${ }^{*}$. F is said to be internal* if it is simultaneously upper and lower internal* .

Definition 7 (C.M.Lee). ([4], [3], p. 35). Let $F:[a, b] \rightarrow \mathbb{R}$. F is said to be $u C M$ if it is increasing on $[c, d] \subseteq[a, b]$, whenever it is so on $(c, d)$. $\mathrm{F}$ is said to be $\ell C M$ if $-F$ is $u C M$. Let $C M=\ell C M \cap u C M$ and $s C M=\{F$ : $F(x)+\lambda x \in C M$ for each $\lambda \in \mathbb{R}\}$. 
Remark 5. ([3], p. 36). Let $F:[a, b] \rightarrow \mathbb{R}$. Then we have:

(i) $\mathcal{C}+$ internal $^{*}=$ internal $^{*}$;

(ii) $\mathcal{C} \subset \mathcal{D B}_{1} \subset \mathcal{D} \subset$ internal $^{*} \subset s C M \subset C M \subset u C M$;

Theorem 1 (Thomson). ( A special case of Theorem 33.1 of [8], p. 74). Let $\mathcal{S}$ be a local system satisfying an intersection condition of the form $\sigma_{x} \cap \sigma_{y} \neq \emptyset$, and let $F:[a, b] \rightarrow \overline{\mathbb{R}}$. If $F$ is $\mathcal{S}$-continuous then $F \in \mathcal{B}_{1}$.

Theorem 2 (Thomson). ([8], $p$. 77). Let $\mathcal{S}$ be a bilateral local system, and let $F:[a, b] \rightarrow \mathbb{R}$. If $F$ is $\mathcal{B}_{1}$ and $\mathcal{S}$-continuous on $[a, b]$ then $F \in \mathcal{D}$ on $[a, b]$.

Proof. See [1] (Theorem 1.1, (1), (2), pp. 8-9).

Theorem 3. ([3], p. 30.) Let $F:[a, b] \rightarrow \mathbb{R}$ and let $\mathcal{S}=\{\mathcal{S}(x)\}_{x \in \mathbb{R}}$ be a local system satisfying the following conditions:

- $\mathcal{S}$ is $\mathcal{S}_{\infty, \infty}$-filtering on $[a, b]$;

- $\sigma_{x} \cap \sigma_{y} \cap(-\infty, x] \neq \emptyset$;

- $\sigma_{x} \cap \sigma_{y} \cap[y,+\infty) \neq \emptyset$;

- $\mathcal{S} D F(x)$ exists (finite or infinite) at each point $x \in[a, b]$.

Then $\mathcal{S} D F(x)$ is $\mathcal{B}_{1}$ on $[a, b]$.

Theorem 4. ([3], p. 149-150). Let $\mathcal{S}$ be a local system $\mathcal{S}_{\infty, \infty}$-filtering, satisfying intersection condition $\sigma_{x} \cap \sigma_{y} \cap[x, y] \neq \emptyset$, and let $F:[a, b] \rightarrow \mathbb{R}$ be $a$ function satisfying the following conditions:

(1) $F \in s C M$ on $[a, b]$;

(2) $\mathcal{S}$-derivative $\mathcal{S} D F(x)$ exists (finite or infinite) at each $x \in[a, b]$ (respectively $x \in[a, b) ; x \in(a, b))$;

(3) $\mathcal{S} D F(x)$ is $\mathcal{B}_{1}$ on $[a, b]$ (respectively $[a, b) ;(a, b)$ ).

Then we have:

(i) $\mathcal{S} D F(x)$ is $\mathcal{D}$ and

(ii) F fulfills the Mean Value Theorem. 
Lemma 3. Let $\mathcal{S}$ be a local system $\mathcal{S}_{\infty, \infty}$-filtering, satisfying intersection condition $\sigma_{x} \cap \sigma_{y} \cap[x, y] \neq \emptyset$. Let $F, G, H:[a, b] \rightarrow \mathbb{R}, H(x)=(F(b)-F(a))$. $G(x)-(G(b)-G(a)) \cdot F(x)$ such that $\mathcal{S} D F(x)$ exists finite or infinite on $(a, b)$, $G^{\prime}$ exists finite on $(a, b)$ and $H \in s C M$ on $[a, b]$. Then there exists $\xi \in(a, b)$ such that

$$
(F(b)-F(a)) \cdot G^{\prime}(\xi)=(G(b)-G(a)) \cdot \mathcal{S} D F(\xi) .
$$

Proof. We have $H(b)=H(a)=F(b) G(a)-G(b) F(a)$. Clearly $\mathcal{S} D H(x)$ exists finite or infinite on $(a, b)$. By Theorem 4, (ii), there exists $\xi \in(a, b)$ such that $\mathcal{S} D H(\xi)=0$. Now the conclusion of our lemma follows immediately.

Corollary 1. Let $\mathcal{S}$ be a local system $\mathcal{S}_{\infty, \infty}$-filtering, satisfying intersection condition $\sigma_{x} \cap \sigma_{y} \cap[x, y] \neq \emptyset$. Let $F, G:[a, b] \rightarrow \mathbb{R}$. If

(i) $F \in$ internal $^{*}$ and $G \in \mathcal{C}$ on $[a, b]$,

(ii) $\mathcal{S}$-derivative $\mathcal{S} D F(x)$ exists finite or infinite on $(a, b)$ and $G^{\prime}(x)$ exists finite on $(a, b)$,

then there exists $\xi \in(a, b)$ such that

$$
(F(b)-F(a)) \cdot G^{\prime}(\xi)=(G(b)-G(a)) \cdot \mathcal{S} D F(\xi) .
$$

Proof. Let $H$ be the function defined in Lemma 3. Since $\mathcal{C}+$ internal $^{*}=$ internal $^{*} \subset s C M$ (see Remark 5) it follows that $H \in s C M$ on $[a, b]$. Now the proof follows by Lemma 3 .

Remark 6. In Lemma 3 and Corollary 1 we may put $\mathcal{S} D G$ instead of $G^{\prime}$ if $\mathcal{S}$ is supposed to be filtering.

Theorem 5. (A strong form of Taylor's Theorem). Let $\mathcal{S}$ be a local system $\mathcal{S}_{\infty, \infty}$-filtering, satisfying the following intersection conditions:

- $\sigma_{x} \cap \sigma_{y} \cap[x, y] \neq \emptyset$;

- $\sigma_{x} \cap \sigma_{y} \cap(-\infty, x] \neq \emptyset$;

- $\sigma_{x} \cap \sigma_{y} \cap[y,+\infty) \neq \emptyset$.

Let $F:[a, b] \rightarrow \mathbb{R}$ such that $F(b-)=F(b)$ if $F(b-)$ exists, and let $n>1$ be an integer. If

(i) $F$ is $\mathcal{S}^{(i)}$-derivable on $[a, b), i=1,2, \ldots, n$ and

(ii) $\mathcal{S D F}^{(n+1)}(x)$ exists finite or infinite on $(a, b)$, 
then there exists $\xi \in(a, b)$ such that

$$
F(b)=\sum_{i=0}^{n} \frac{\mathcal{S} D F^{(i)}(a)}{i !}(b-a)^{i}+\frac{\mathcal{S} D F^{(n+1)}(\xi)}{(n+1) !}(b-a)^{n+1} .
$$

Proof. Let

$$
R(x)=F(x)-\sum_{i=0}^{n} \frac{\mathcal{S} D F^{(i)}(a)}{i !}(x-a)^{i} \text { and } G(x)=(x-a)^{n+1} .
$$

Clearly $R(a)=\mathcal{S} D R(a)=\ldots=\mathcal{S} D R^{(n)}(a)=0$ and $\mathcal{S} D R^{(n+1)}(x)=$ $\mathcal{S} D F^{(n+1)}(x)$ for each $x \in(a, b)$. But $G(a)=G^{\prime}(a)=\ldots=G^{(n)}(a)=0$ and $G^{(n+1)}(x)=(n+1)$ ! on $(a, b)$. By Theorem $3, \mathcal{S} D F^{(i)}$ is $\mathcal{B}_{1}$ on $[a, b)$, $i=1,2, \ldots, n$ and $\mathcal{S} D F^{(n+1)}$ is $\mathcal{B}_{1}$ on $(a, b)$. By Theorem 4 , (i) it follows that $\mathcal{S} D F^{(i)} \in \mathcal{D}$ on $[a, b), i=1,2, \ldots, n$, and $\mathcal{S} D F^{(n+1)} \in \mathcal{D}$ on $(a, b)$. By Lemma $2, F$ is $\mathcal{S}$-continuous on $[a, b)$, so by Theorem $1, F \in \mathcal{B}_{1}$ on $[a, b)$. By Theorem $2, F \in \mathcal{D}$ on $[a, b)$. By Remark 5, (ii) and the fact that $F(b-)=F(b)$ if $F(b-)$ exists, it follows that $F \in$ internal $^{*}$ on $[a, b]$. Then $R \in$ internal $^{*}$ on $[a, b]$ (see Remark 5, (i)). Applying Corollary 1, it follows that there exists $c_{1} \in(a, b)$ such that $R(b) / G(b)=\mathcal{S} D F\left(c_{1}\right) / G^{\prime}\left(c_{1}\right)$. Since $\mathcal{S} D F \in \mathcal{D B}_{1} \subset$ internal $^{*}$ on $\left[a, c_{1}\right]$ (see Remark 5), applying Corollary 1 again, it follows that there exists $c_{2} \in\left(a, c_{1}\right)$ such that $\mathcal{S} D F\left(c_{1}\right) / G^{\prime}\left(c_{1}\right)=\mathcal{S} D F^{(2)}\left(c_{2}\right) / G^{(2)}\left(c_{2}\right)$. Continuing, we obtain $b>c_{1}>c_{2}>\ldots>c_{n}>c_{n+1}>a$ such that

$$
\begin{gathered}
\frac{R(b)}{G(b)}=\frac{\mathcal{S} D R\left(c_{1}\right)}{G^{\prime}\left(c_{1}\right)}=\ldots=\frac{\mathcal{S} D R^{(n)}\left(c_{n}\right)}{G^{(n)}\left(c_{n}\right)}= \\
\frac{\mathcal{S} D R^{(n+1)}\left(c_{n+1}\right)}{(n+1) !}=\frac{\mathcal{S} D F^{(n+1)}\left(c_{n+1}\right)}{(n+1) !} .
\end{gathered}
$$

Putting $\xi=c_{n+1}$ the assertion of the theorem follows.

Corollary 2. Let $F:[a, b] \rightarrow \mathbb{R}$ and let $n \geq 1$ be an integer. Suppose that

(1) $F(b-)=F(b)$ if $F(b-)$ exists;

(2) $F_{a p}^{(i)}(x)$ (respectively $F_{p r}^{(i)}(x)$ ) exists and is finite on $[a, b)$, for each $i=$ $1,2, \ldots, n$ and

(3) $F_{a p}^{(n+1)}(x)$ (respectively $\left.F_{p r}^{(n+1)}(x)\right)$ exists finite or infinite on $(a, b)$. 
Then there exists $\xi \in(a, b)$ such that

$$
F(b)=\sum_{i=0}^{n} \frac{F_{a p}^{(i)}(a)}{i !}(b-a)^{i}+\frac{F_{a p}^{(n+1)}(\xi)}{(n+1) !}(b-a)^{n+1}
$$

(respectively

$$
\left.F(b)=\sum_{i=0}^{n} \frac{F_{p r}^{(i)}(a)}{i !}(b-a)^{i}+\frac{F_{p r}^{(n+1)}(\xi)}{(n+1) !}(b-a)^{n+1}\right) .
$$

\section{References}

[1] A. M. Bruckner, Differentiation of real functions, Lect. Notes in Math., vol. 659, Springer-Verlag, 1978.

[2] A. M. Bruckner, R. J. O'Malley, and B. S. Thomson, Path derivatives: a unified view of certain generalized derivatives, Trans. Amer. Math. Soc. 283 (1984), 97-125.

[3] V. Ene, Real functions - current topics, Lect. Notes in Math., vol. 1603, Springer-Verlag, 1995.

[4] C. M. Lee, An analogue of the theorem Hake-Alexandroff-Looman, Fund. Math. C (1978), 69-74.

[5] D. Preiss, Approximate derivatives and Baire classes, Czech. Math. J. 21 (1971), no. $96,373-382$.

[6] B. S. Thomson, On full covering properties, Real Analysis Exchange 6 (1980-81), no. 1, 77-93.

[7] B. S. Thomson, Derivation bases on the real line, I and II, Real Analysis Exchange 8 (1982-1983), 67-208 and 280-442.

[8] B. S. Thomson, Real functions, Lect. Notes in Math., vol. 1170, SpringerVerlag, 1985. 